\title{
A releuância da localização para a recuperação de informações visuais
}

\section{La releuancia de la Localización para la recuperación de información vísual The Releuance of Location for Retrieuing Uisual Information}

\author{
Lorena Barbosa Cunha Macedo \\ Cesar Galera \\ Universidade de São Paulo
}

Doi: https://doi.org/10.12804/revistas.urosario.edu.co/apl/a.9876

\section{Resumo}

O papel da localização na integração das características visuais na memória é controverso. Algumas evidências sugerem que a localização perde importância logo depois da consolidação da informação, outras, que a localização permanece associada à representação do objeto por mais tempo. Nós investigamos o papel da localização para a memória de objetos definidos por cor e forma. Os participantes (36) realizaram uma tarefa de deteç̧ão de mudança com 3 e 6 estímulos, com dois intervalos de retenção (500 e 1.500 milissegundos). O desempenho é melhor quando a carga é menor, quando os estímulos são apresentados nos mesmos lugares em que foram memorizados, e depende da interação entre estes fatores. $\mathrm{O}$ intervalo de retenção não interfere no efeito da localização, mas modula o efeito da carga. Estes resultados sugerem que a localização é codificada de forma incidental com os estímulos visuais e que esta associação permanece inalterada dentro dos limites de tempo investigados. Palavras-chave: localização; conjunção cor-forma; memória visual.

\section{Resumen}

El papel de la localización en la integración de las características visuales en la memoria es controvertido. Algunas evidencias sugieren que la localización pierde importancia poco después de la consolidación de la información, otras, que la localización permanece asociada a la representación del objeto durante más tiempo. Investigamos el papel de la localización para la memoria de los objetos definidos por el color y la forma. Los 36 participantes realizaron una tarea de detección de cambios con 3 y 6 estímulos, con dos intervalos de retención (500 y 1.500 milisegundos). El rendimiento es mejor cuando la carga es menor, cuando

Lorena Barbosa Cunha Macedo ORCID ID: https://orcid.org/0000-0001-8497-2162

Cesar Galera ORCID ID: https://orcid.org/0000-0002-3021-7126

O presente trabalho foi realizado com apoio da Coordenação de Aperfeiçoamento de Pessoal de Nível Superior (CAPES) - Brasil - Código de Financiamento 001. Conselho Nacional de Desenvolvimento Científico (CNPQ).

Dirigir correspondência à Lorena Barbosa Cunha Macedo. Endereço: Av. Bandeirantes, 3900, CEP: 14049-900, Brasil. Correio eletrônico: lorenamacedo@usp.br

Para citar este artigo: Macedo, L. B. C., \& Galera, C. (2021). A relevância da localização para a recuperação de informações visuais. Avances en Psicología Latinoamericana, 39(1), 1-9. https://doi.org/10.12804/revistas.urosario.edu.co/apl/a.9876 
los estímulos se presentan en los mismos lugares donde fueron memorizados, y depende de la interacción entre estos factores. El intervalo de retención no interfiere con el efecto de ubicación, pero modula el efecto de carga. Estos resultados sugieren que la ubicación se codifica incidentalmente con estímulos visuales y que esta asociación permanece sin cambios dentro de los límites de tiempo investigados.

Palabras clave: localización; conjunción color-forma; memoria visual.

\section{fbstract}

The role of localization in the integration of visual characteristics into memory is controversial. Some evidence suggests that location loses importance soon after the consolidation of information; others, that the location remains associated with the representation of the object for longer. We investigated the role of location for the memory of objects defined by color and shape. Participants (36) performed a change detection task with 3 and 6 stimuli, with two retention intervals (500 and 1.500 milliseconds). Performance is better when the load is lower, stimuli are presented in the same places where they were memorized, and it depends on the interaction between these factors. The retention interval does not interfere with the location effect but modulates that of the load. These results suggest that localization is incidentally encoded with visual stimuli and that this association remains unchanged within the time limits investigated. Keywords: Location; color-shape conjunction; visual memory.

A localização dos objetos no espaço tem um papel central para a integração de características visuais na percepção. Durante a percepção, as características visuais primitivas como cor, forma, orientação, e movimento, são codificadas por diferentes receptores visuais e processadas em áreas corticais distintas (Hubel \& Wisel, 1962). De acordo com a Teoria de Integração de Características (TIC), a percepção de um objeto íntegro tal como se apresenta à nossa consciência, resulta de um processo de integração das características codificadas nas diferentes áreas do córtex visual.

Este processo de integração depende da focalização da atenção numa representação do espaço que contém a informação sobre localizações dos objetos, mas não sobre as características visuais que os compõem. A atenção focalizada nos locais ocupados pelos objetos, age como uma "cola" que integra de forma coerente as características ali referenciadas, mas codificadas em outras regiões corticais (Treisman \& Gelade, 1980). A informação sobre localização de um objeto é assim, um pré-requisito para que suas características visuais sejam integradas na forma de um objeto disponível à percepção consciente.

Quando aplicada ao estudo da memória, a TIC leva de maneira imediata à questão da importância da localização para o armazenamento e para a recuperação dos objetos. Segundo Treisman e colaboradores (Kahneman \& Treisman, 1984; Treisman \& Zhang, 2006; Wheeler \& Treisman, 2002) a representação episódica de um objeto, identificada como um arquivo de objeto (object file), é uma representação temporária, que contém a aparência visual do objeto, assim como sua localização e distância em relação a outros objetos e ao sujeito.

A localização é uma característica chave da representação memorizada, pois é através da localização do objeto no espaço, que sua representação pode ser acessada e a atualizada. A contribuição da localização para a recuperação da informação visual ocorre tanto para objetos familiares, quanto para objetos não familiares ao indivíduo (Kovacs \& Harris, 2019), sendo mais efetiva para características apresentadas agrupadas ao invés de isoladas (Treisman \& Zhang, 2006), ou quando são apresentadas de forma simultânea ao invés de sequencial (Bharti et al., 2020).

Estudos sobre a memória para objetos em cenas naturais, mostram também que a recuperação da informação de um objeto é fortemente facilitada por sua localização. Hollingworth e Henderson 
(2002), por exemplo, mostraram que olhar para a localização da cena em que estava um objeto facilita sua recuperação. Ou ainda, dirigir a atenção para o local em que um objeto foi apresentado durante a memorização, produz uma melhora significativa no desempenho de uma tarefa de reconhecimento (Griffing \& Nobre, 2003). Resultados equivalentes têm sido obtidos em modelos que simulam o funcionamento neural da memória humana com base no desempenho em tarefas de recordação indicada (cued recall) que utilizam o espaço como dica de recuperação. De acordo com Schneegans e Bays (2017), resultados comportamentais obtidos em tarefas de recordação, são consistentes com um modelo em que as características visuais não espaciais são integradas exclusivamente por meio da localização que partilham, reafirmando a importância da localização na conjunção de características visuais, como cor e forma.

No entanto, alguns estudos sugerem que o papel da localização para a memória visual é restrito à codificação e aos estágios iniciais de consolidação da informação na memória. Evidências nesse sentido foram obtidas em estudos que utilizam tarefas nas quais os participantes são instruídos a memorizar um ou mais objetos distribuídos numa tela e, depois de um intervalo de retenção, frente a apresentação de uma tela teste, são instruídos a decidir se os estímulos presentes no teste são iguais aos memorizados, independentemente do local em que foram apresentados.

Os resultados obtidos com esse tipo de paradigma experimental, mostram que existe uma vantagem quando os estímulos são iguais e apresentados nos mesmos lugares, do que quando são iguais, mas apresentados em lugares diferentes. Esta vantagem acontece quando o intervalo de retenção é curto, em torno de 100 milissegundos (ms) e tende a desaparecer em intervalos superiores a $1.000 \mathrm{~ms}$, ou seja, a localização seria importante para a memória dos objetos visuais nos estágios iniciais da consolidação desses objetos na memória, e perderia sua importância em inter- valos de retenção mais longos. Treisman e Zhang (2006), por exemplo, em um estudo no qual os participantes memorizaram três figuras definidas pela conjunção de cor e forma, encontraram uma contribuição significativa da localização, que tende a desaparecer em torno de $1.000 \mathrm{~ms}$ (exp. 3), quando apenas dois intervalos de retenção foram examinados (100 ms e $900 \mathrm{~ms}$ ), mas que pode se estender até $3.000 \mathrm{~ms}$ (exp. 5) quando o intervalo de retenção varia de $100 \mathrm{~ms}$ a $6.000 \mathrm{~ms}$.

Resultados semelhantes foram obtidos por Logie et al. (2011), com uma carga de memória de seis figuras, também definidas pela conjunção de cor e forma, e intervalos de retenção variando de 0 a 2.500 ms. Nesse estudo de Logie et al. (2011), a localização parece contribuir para a recuperação da informação memorizada por um intervalo de tempo menor do que $1.500 \mathrm{~ms}$. Por outro lado, alguns estudos sugerem que a informação sobre a localização seria codificada de forma incidental com a informação visual de cores, e poderia interferir em seu reconhecimento em intervalos de retenção que chegam a $6.000 \mathrm{~ms}$ (Olson \& Marshuetz, 2005) ou mesmo a 15.000 ms (Elsley \& Parmentier, 2015).

As diferenças entre os resultados obtidos nos estudos apresentados acima, podem ser atribuídas às diferenças entre os procedimentos experimentais empregados, principalmente à carga de memória e os intervalos de retenção nos experimentos de Treisman e Zhang (2006) e de Logie et al. (2011). A diferença na carga de memória pode, por exemplo, interferir no tempo necessário à consolidação dos estímulos na memória, ou seja, depois que as características visuais isoladas foram integradas em um objeto coerente, e consolidadas em representações mais abstratas e duradouras na memória. De acordo com Vogel et al. (2006), este processo de consolidação depende do número de objetos a serem memorizados e acontece a uma taxa média de $50 \mathrm{~ms}$ por item memorizado.

Dessa forma, considerou-se relevante investigar o papel da localização para a memória visual de curto prazo manipulando a carga de memória 
e o intervalo de retenção, utilizando uma tarefa de detecção de mudança, a mesma utilizada por Treisman e Zhang (2006) e por Logie et al. (2011).

Neste estudo foi investigada a contribuição da localização para a recuperação da informação visual armazenada na memória de trabalho visual. A suposição inicial era que se a localização perde sua importância depois que os estímulos são consolidados na memória, este processo seria mais rápido quando a carga de memória fosse menor, uma vez que o tempo de consolidação varia em função do número de estímulos a serem memorizados. Assim como em Logie et al. (2011) e Treisman e Zhang (2006), foi utilizada uma tarefa de detecção de mudança, na qual os participantes memorizaram um conjunto de estímulos definidos pela conjunção de cor e forma e, depois de um intervalo de retenção, deveriam decidir se um conjunto de estímulos apresentados como teste eram iguais ou diferentes daqueles memorizados, independentemente da localização em que eram apresentados.

\section{Método}

\section{Participantes}

O estudo contou com 36 voluntários ( 25 mulheres), com idades entre 18 e 35 anos ( $M=25.25$ anos, $D P=3.93$ ), com visão normal ou corrigida. Este estudo foi aprovado pelo Comitê de Ética em Pesquisa com seres humanos da Universidade de São Paulo (CAAE n. ${ }^{\circ}$ 79030817.0.0000.5407), e todos os participantes assinaram o termo de consentimento livre e esclarecido.

\section{Material e estímulos}

Os estímulos memorizados foram definidos pela conjunção de seis cores (verde, vermelho, azul, amarelo, laranja e lilás) e seis formas (círculo, estrela, cruz, losango, quadrado e triângulo), sendo que nenhuma cor ou forma foram repetidos em uma mesma prova. Os estímulos foram apresentados ao redor do ponto central da tela com resolução 1920 x 1080 pixels, em uma matriz quadrada de $3 \times 3$ células, cada uma medindo 94 x 94 pixels. Metade dos participantes realizou prova com a apresentação de 3 estímulos, e a outra metade, provas com a apresentação de 6 estímulos.

\section{Procedimento}

Os participantes realizaram uma tarefa de detecção de mudança, na qual deveriam memorizar uma cena com figuras coloridas definidas pela conjunção de cor e forma, e em seguida, depois do intervalo de retenção, julgar se a cena teste apresentava exatamente as mesmas figuras da cena inicial. O número de estímulos memorizados (três ou seis) foi manipulado entre participantes, sendo cada grupo com 18 participantes. Os participantes eram informados no início da sessão que a localização das figuras era irrelevante para a tarefa, e que poderia mudar da cena memorizada para a cena teste. Durante a prova os participantes realizaram uma tarefa de supressão articulatória que consistia na repetição em voz alta de três dígitos.

Cada prova (figura 1) teve início com a apresentação de tela de fixação por $1.000 \mathrm{~ms}$, seguidos pela apresentação por $100 \mathrm{~ms}$ dos números da supressão articulatória. A tela com os estímulos a serem memorizados foi apresentada em seguida por $200 \mathrm{~ms}$, seguida do IR (500 ms ou $1.500 \mathrm{~ms}$ ), depois do qual foi apresentada a tela teste, que permaneceu até que o voluntário desse a sua resposta. Respostas "sim" (houve alteração, ou seja, houve uma troca de cores em relação as formas memorizadas), foram dadas na tecla do lado direito do mouse. Respostas "não" (não houve mudança, estímulos iguais aos memorizados), foram dadas na tecla esquerda do mouse. Um feedback sobre a precisão da resposta foi apresentado ao final de cada prova. Esse procedimento foi igual tanto para os participantes que memorizaram 3 estímulos, quanto para os que memorizaram 6 estímulos. 


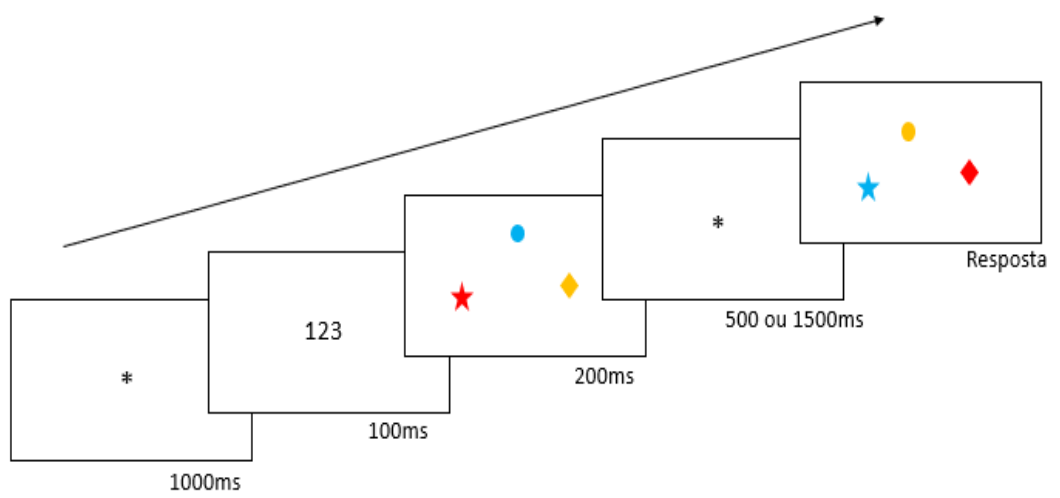

Figura 1. Representação esquemática do experimento, para condição de 3 estímulos a serem memorizados. No exemplo, o participante deveria pressionar a tecla direita do mouse (houve mudança, ou seja, houve troca de cores entre as formas memorizadas)

Foram realizadas 160 provas na sessão experimental, sendo que em 80 delas, o IR foi de $500 \mathrm{~ms}$, e de 1.500 ms nas demais. Em $50 \%$ das provas os estímulos apresentados para memorização foram iguais (resposta "igual") aos apresentados na tela teste. Independentemente do tipo de resposta, em metade das provas as figuras foram apresentadas na tela teste nas mesmas localizações ocupadas na tela inicial (mesma localização), e na outra metade, elas ocupavam localizações diferentes das apresentadas na tela inicial (localização nova). Nas provas em que a localização mudou, a posição das figuras coloridas mudou de maneira aleatória entre as caselas da matriz de localização. Os tratamentos resultantes da combinação de IR, tipo de resposta (igual ou diferente) e do tipo de localização foram manipulados aleatoriamente na sessão.

\section{Resultados}

As respostas de cada participante (hits e alarmes falsos), obtidas nos diferentes tratamentos experimentais, foram convertidas em índices de discriminação (d') (Wickens, 2002; Snodgrass \& Corwin, 1988). Os $d$ ' foram submetidos a uma análise de variância (ANOVA) que levou em conta o número de estímulos memorizados (três e seis) como fator manipulado entre participantes, as medidas repetidas nos tratamentos resultantes da combinação do intervalo de retenção (500 ms e $1.500 \mathrm{~ms}$ ) e mudança na localização (mesma localização, localização nova). As médias dos tempos de reação (TRs), estimadas sem levar em conta os TRs das provas com respostas incorretas, e os TRs abaixo de $300 \mathrm{~ms}$ e acima de 5.000 ( $0.35 \%$ das respostas $)$ não foram submetidos à mesma ANOVA que os $d$ 's.

Os resultados revelam que a carga de estímulos memorizados e a mudança da localização no teste, são os principais determinantes do desempenho. $\mathrm{O}$ intervalo de retenção tem um papel secundário, associado à carga, mas não à mudança de localização dos estímulos. O índice de discriminação é melhor com três estímulos $(M=1.74, E P M=0.08)$ do que com seis $(M=0.88, E P M=0.08), F(1.34)=49.38, p$ $<.001 ; \eta^{2}=0.59$. O desempenho foi melhor nas provas em que os estímulos foram apresentados nas mesmas localizações $(M=1.62, E P M=0.11)$ do que quando foram apresentados em localizações novas $(M=0.99, E P M=0.09), F(1.34)=84.87, p<.001$, $\eta^{2}=0.71$. Estes dois fatores, carga e localização, tem efeitos combinados significativos, $F(1.34)=22.43$, $p<.001, \eta^{2}=0.40$. A vantagem proporcionada pela apresentação dos estímulos na mesma localização, estimada pela diferença nas duas condições do fator localização, é maior quando a carga é de três estímulos $(M=0.98, E P M=0.1)$ do que quando a carga é de seis estímulos $(M=0.31, E P M=0.01)$. 


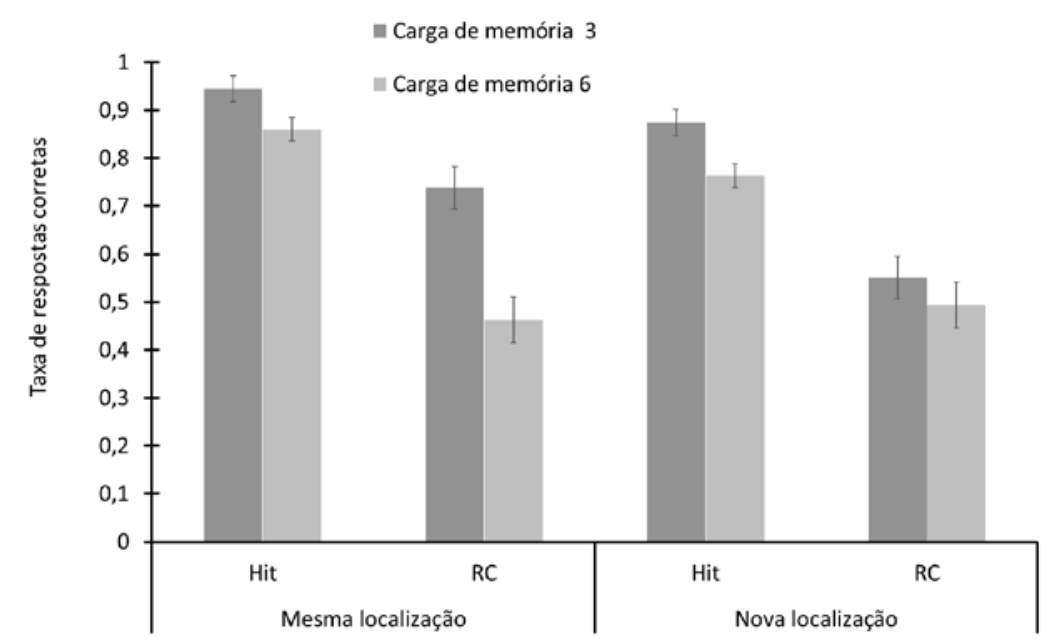

Figura 2. Taxa de respostas corretas para respostas positivas (Hit) e rejeições corretas (RC) em função da posição do teste para as cargas de memória de 3 e 6 itens. As barras verticais representam o erro padrão da média

A variação do $d$ ' em função do intervalo de retenção não foi significativa, ou seja, não existe uma diminuição significativa no desempenho em função do intervalo de retenção, $F(1.34)=2.82$, $p=0.10, \eta^{2}=0.07$. Este fator tem um efeito significativo apenas na interação com a carga de estímulos, $F(1.34)=4.48, p=0.04, \eta^{2}=0.12$. Nas provas com três estímulos, o desempenho não varia em função da duração do intervalo de retenção $(p=1)$, mas nas provas com carga de 6 estímulos, o desempenho é melhor no intervalo mais curto do que no mais longo $(p=0.03)$. E, mais importante ainda para os objetivos deste estudo, a interação entre o intervalo de retenção e a mudança na localização dos estímulos no teste não é significativa $(F<1)$, o que sugere que a localização permanece relevante para a recuperação da informação memorizada mesmo em intervalos de tempo mais longos.

Foi avaliada também a possibilidade de que o desempenho obtido nos diferentes tratamentos experimentais, principalmente naqueles com desempenho mais prejudicado, possa ter chegado ao nível do acaso. Isso foi feito através de testes $t$, nos quais avaliou-se a probabilidade de o desempenho ser igual a zero. Essa análise mostra que o $d$ ' foi maior do que zero em todos os tratamentos realizados $(p<0.001)$.

Também existe um custo temporal significativo quando os estímulos são apresentados em locais diferentes $\left.F(1.34)=94.52, p<.001, \eta^{2}=0.74\right)$. O TR para decidir que os estímulos são iguais, é menor quando os estímulos são apresentados na mesma localização $(1001 \mathrm{~ms}, \mathrm{EPM}=51 \mathrm{~ms})$ do que quando são apresentados em locais diferentes ( $\mathrm{TR}=1156 \mathrm{~ms}, \mathrm{EPM}=57 \mathrm{~ms}$ ). $\mathrm{O}$ TR não é afetado por nenhum outro fator ou interação entre fatores.

\section{Discussão}

Neste estudo avaliou-se a contribuição da informação de localização para a recuperação de objetos formados pela conjunção de cor e forma. A localização exerce um papel relevante na integração das diferentes características visuais desde o armazenamento até a recuperação (Treisman \& Gelade, 1980). Enquanto alguns estudos sugerem que em tarefas de memória sua importância parece diminuir após a consolidação das características visuais na memória de trabalho visual (Logie et al., 2011; Treisman \& Zhang, 2006), e que a 
vantagem obtida nas provas em que, no teste, os estímulos são apresentados nos mesmos lugares tende a desaparecer com intervalos de retenção mais longos (Logie et al., 2011). Outros estudos sugerem que a localização permanece por mais tempo como um fator importante para a memória da conjunção de elementos visuais (Elsley \& Parmentier, 2015; Olson \& Marshuetz, 2005). Nesses estudos, a contribuição da localização é estimada comparando-se o desempenho em situações nas quais, no teste, os estímulos são apresentados nos mesmos locais em que haviam sido memorizados, ou em locais diferentes.

Os resultados obtidos neste estudo mostram que, a vantagem encontrada quando os estímulos são apresentados nos mesmos lugares em que foram memorizados independe do intervalo de retenção. Ou seja, a localização é codificada de forma incidental, e permanece importante para o processo de recuperação mesmo quando o intervalo de retenção é mais longo. Na codificação incidental há uma junção de informações, sem que haja um esforço voluntário para tal. É o que ocorre por exemplo com a localização em relação a informação de cor, como mostrado por Jiang et al. (2000), na qual os autores verificaram que mudanças na localização do objeto memorizado interferem na recuperação da informação de cor, mas o contrário não é verdadeiro. Olson e Marshuetz (2005) propõem que a informação de localização sempre estará contida junto ao item memorizado, de maneira inerente à sua memorização.

Logie et al. (2011) e Treisman e Zhang (2006) indicam que a manutenção da localização auxilia na recuperação dos itens, pois funciona como uma dica visual que auxilia nesse processo. A diferença deste estudo, em relação ao dos referidos autores, encontra-se no intervalo de tempo no qual a localização continua a auxiliar na recuperação dos estímulos. Nos estudos de Logie et al. (2011) e Treisman e Zhang (2006), a vantagem da locali- zação se perde a partir de intervalos entre $900 \mathrm{~ms}$ e $1.000 \mathrm{~ms}$, enquanto no presente estudo a localização contribuiu para a recuperação mesmo em $1.500 \mathrm{~ms}$.

A permanência da localização por períodos mais extensos é evidente também no tempo de resposta, que aumenta de forma significativa nas provas em que os estímulos são apresentados em locais diferentes daquele nos quais foram memorizados. De acordo com Treisman e Schmidt (1982), o tempo extra para reconhecimento dos estímulos iguais apresentados em locais diferentes, indica o custo de reorganizá-los no espaço, devido ao mismatch em relação a cena inicial, ou é possível chamar também de uma conjunção ilusória, devido a alteração da localização associada ao objeto memorizado. Neste caso a localização funciona como uma dica, que facilita a recuperação dos estímulos, reduzindo o tempo necessário na tomada de decisão em tarefas de detecção de mudança.

Os resultados deste estudo mostram que a vantagem proporcionada pela localização, depende da carga da memória. A taxa de acerto foi maior para a carga de 3 objetos, do que para 6 objetos. Embora muitas dúvidas permaneçam a respeito do armazenamento das características visuais que compõem um objeto, Luck e Vogel (1997) propõem que o sistema responsável pelo armazenamento de informações visuais teria capacidade para armazenar apenas 4 objetos complexos, formados por diferentes características visuais. Partindo desse pressuposto, é possível supor que a diminuição do índice de discriminação obtido com o aumento da carga de 3 para 6 objetos, pode ser ocasionada pela limitação do sistema no armazenamento da carga extra. Desse modo, por estar com sua capacidade de armazenamento completa, o sistema não teria recursos disponíveis para armazenamento dos itens em excesso, prejudicando sua recuperação. Mas, mesmo para carga de 6 objetos, o índice de discriminação continuou indicando um desempenho superior ao acaso. 


\section{Considerações finais}

O objetivo desse estudo foi verificar a contribuição da localização para a recuperação de objetos formados pela conjunção de cor e forma. Verificou-se que apresentar os estímulos na cena teste no mesmo local em que foram memorizados, favoreceu a sua recuperação tanto em intervalos de $500 \mathrm{~ms}$ quanto de $1.500 \mathrm{~ms}$. Diferente de Treisman e Zhang (2006) e Logie et al. (2011), o presente estudo verificou que a carga de itens memorizados parece um fator de maior interferência no papel da localização para a memória de trabalho visual (MTV), do que os intervalos de retenção.

O intervalo de tempo pelo qual a informação de localização favorece a recuperação das informações consolidadas na MTV, permanece ainda controverso. Apesar disso, os resultados sugerem que a quantidade de objetos memorizados parece estar relacionada a essa relevância, merecendo assim futuras explorações.

\section{Referências}

Bharti, A. K., Yadav, S. K., \& Jaswal, S. (2020). Feature binding of sequentially presented stimuli in visual working memory. Frontiers in Psychology, 11, 33. https://doi.org/10.3389/ fpsyg.2020.00033

Elsley, J. V., \& Parmentier, F. B. R. (2015). The asymmetry and temporal dynamics of incidental letter-location bindings in working memory. Quarterly Journal of Experimental Psychology, 68(3), 433-441. https://doi.org/10.1080/1747 0218.2014.982137

Griffin, I. C., \& Nobre, A. C. (2003). Orienting attention to locations in internal representations. Journal of Cognitive Neuroscience, 15, 1176-1194. https://doi.org/10.1162/089892903322598139

Hollingworth, A., \& Henderson, J. M. (2002). Accurate visual memory for previously attended objects in natural scenes. Journal of Experimental Psy- chology: Human Perception and Performance, 28(1), 113-136. https://doi.org/10.1037/00961523.28.1.113

Hubel, D. H., \& Wiesel, T. N. (1962). Receptive fields, binocular interaction and functional architecture in the cat's visual cortex. The Journal of Physiology, 160, 106-154. https://doi. org/10.1113/jphysiol.1962.sp006837

Kahneman, D., \& Treisman, A. (1984). Changing views of attention \& automaticity. In R. Parasuraman \& D. R. Davies (Eds.), Varieties of attention (pp. 29-61). Academic Press. https:// ci.nii.ac.jp/naid/10018424113/

Kovacs, O., \& Harris, I. M. (2019). The role of location in visual feature binding. Attention, Perception, and Psychophysics, 81, 1551-1563. https://doi.org/10.3758/s13414-018-01638-8

Jiang, Y., Olson, I. R., \& Chun, M. M. (2000). Organization of visual short-term memory. Journal of Experimental Psychology: Learning, Memory, and Cognition, 26(3), 683-702. http://doi. org/10.1037/0278-7393.26.3.683

Logie, R. H., Brockmole, J. R., \& Jaswal, S. (2011). Feature binding in visual short-term memory is unaffected by task-irrelevant changes of location, shape, and color. Memory \& Cognition, 39(1), 24-36. https://doi.org/10.3758/s13421010-0001-z

Luck, S. J., \& Vogel, E. K. (1997). The capacity of visual working memory for features and conjunctions. Nature, 390, 279-281. https://doi. org $/ 10.1038 / 36846$

Olson, I. R., \& Marshuetz, C. (2005). Remembering "what" brings along "where" in visual working memory. Perception \& Psychophysics, 67(2), 185-194. https://doi.org/10.3758/BF03206483

Schneegans, S., \& Bays, P. M. (2017). Neural architecture for feature binding in visual working memory. The Journal of Neuroscience, 37(14), 3913-3925. https://doi.org/10.1523/JNEUROSCI.3493-16.2017

Snodgrass, G., \& Corwin, J. (1988). Pragmatics of measuring recognition memory: Applications 
to dementia and amnesia. Journal of Experimental Psychology: General, 117(1), 34-50.

Treisman, A., \& Gelade, G. (1980). A feature-integration theory of attention. Cognitive Psychology, 12, 97-136. https://doi.org/10.1016/00100285(80)90005-5

Treisman, A., \& Schmidt, H. (1982). Illusory conjunctions in the perception of objects. Cognitive Psychology, 14(1), 107-141. https://doi. org/10.1016/0010-0285(82)90006-8

Treisman, A., \& Zhang, W. (2006). Location and binding in visual working memory. Memory \& Cognition, 34(8), 1704-1719. https://doi. org/10.3758/BF03195932
Vogel, E. K., Woodman, G. F., \& Luck, S. J. (2006). The time course of consolidation in visual working memory. Journal of Experimental Psychology: Human Perception and Performance, 32(6), 1436-1451. https://doi.org/10.1037/00961523.32.6.1436

Wheeler, M. E., \& Treisman, A. M. (2002). Binding in short-term visual memory. Journal of Experimental Psychology: General, 131(1), 48-64. https://doi.org/10.1037//0096-3445.131.1.48

Wickens,T. D. (2002). Elementary signal detection theory. Oxford University Press. https:// doi.org/10.1093/acprof:oso/9780195092509. 001.0001

\section{Recebido: novembro 12, 2020 Aprovado: julho 28, 2021}

